\title{
Pulmonary Artery Involvement in Patients with Takayasu Arteritis
}

\author{
Yanru He, Naqiang Lv, Aimin Dang, and Nan Cheng
}

ABSTRACT. Objective. This study was performed to explore the clinical manifestations and longterm prognosis in patients with Takayasu arteritis (TA) with pulmonary artery involvement (PAI).

Methods. The medical records of 194 patients with TA who underwent traditional catheter angiography or computed tomography of pulmonary artery from 2009 to 2016 were retrospectively reviewed. The clinical manifestations, angiographic features, and mortality of 128 patients with TA with PAI were further analyzed.

Results. Patients with TA with PAI had a higher risk of pulmonary hypertension (PH) than patients with TA alone $(61.7 \%$ vs $7.6 \%, \mathrm{p}<0.001)$. Patients with PAI and PH more frequently developed dyspnea, hemoptysis, and lower limbs edema (all $\mathrm{p}<0.05$ ) than those without PH. Patients with PH also had a higher incidence of bilateral PAI $(84.8 \%$ vs $34.7 \%, \mathrm{p}<0.001)$ and a higher pulmonary artery obstruction index [23 (interquartile range 20-27) vs 10 (6-15), p < 0.001]. Left heart disease was presented in $39(30.5 \%)$ patients with TA with PAI. During the median followup of 38 (21-58) months, 19 and 2 deaths occurred among patients with and without $\mathrm{PH}$, respectively. Among patients with PAI, the mortality rate was 7 times higher in patients with than without PH $(\mathrm{p}=0.009)$. Independent predictors of mortality were the disease duration $(p=0.047)$, New York Heart Association class III/IV $(\mathrm{p}=0.019)$, right ventricular systolic dysfunction $(\mathrm{p}=0.019)$, and respiratory failure $(\mathrm{p}=$ 0.007).

Conclusion. Patients with TA with PAI have a higher risk of developing PH than patients with TA alone. The presence of PH in patients with PAI increases the risk of early mortality. (First Release October 15 2019; J Rheumatol 2020;47:264-72; doi:10.3899/jrheum.190045)

\section{Key Indexing Terms: \\ TAKAYASU ARTERITIS PULMONARY HYPERTENSION}

Takayasu arteritis (TA) is a chronic granulomatous vasculitis that primarily affects the aorta and its main branches, including the pulmonary artery $(\mathrm{PA})^{1,2,3}$. The profound inflammatory process in the affected arteries can lead to stenosis, occlusion, aneurysms, and end-organ ischemia, thereby increasing the risk of cardiovascular morbidity and mortality.

From the Fuwai Hospital, National Clinical Research Center for Cardiovascular Diseases, Chinese Academy of Medical Sciences (CAMS) and Peking Union Medical College, Beijing, China.

This study is supported by the National Natural Science Foundation of China (grant no. 30770859 and 81170285) and CAMS Innovation Fund for Medical Science (CIFMS; 2017-12M-2-002).

Y. He, MD, Fuwai Hospital, National Clinical Research Center for Cardiovascular Diseases, CAMS and Peking Union Medical College; N. Lv, MD, PhD, Fuwai Hospital, National Clinical Research Center for Cardiovascular Diseases, CAMS and Peking Union Medical College; A. Dang, MD, PhD, Fuwai Hospital, National Clinical Research Center for Cardiovascular Diseases, CAMS and Peking Union Medical College; N. Cheng, MD, Fuwai Hospital, National Clinical Research Center for Cardiovascular Diseases, CAMS and Peking Union Medical College.

Address correspondence to Dr. A. Dang, Fuwai Hospital, National Clinical Research Center for Cardiovascular Diseases, Chinese Academy of Medical Sciences and Peking Union Medical College, 167 Beilishi Road, Beijing 100037, China.

E-mail: amdangfw@163.com,amdang@fuwaihospital.org

Accepted for publication May 6, 2019.

\section{PULMONARY ARTERY PROGNOSIS}

PA involvement (PAI) is frequent, occurring in 20-56\% of patients with TA, according to autopsy reports 4,5 However, the pulmonary circulation is often overlooked in the initial assessment of patients with TA. Pulmonary hypertension $(\mathrm{PH})$ is a major complication in the course of TA-associated $\mathrm{PAI}^{6-12}$. The diagnosis of $\mathrm{PH}$ carries a 7-fold higher risk of early mortality in many different clinical conditions $^{13}$. Because of the rarity of the disease, few studies have comprehensively investigated the clinical manifestations and longterm outcomes of TA with PAI.

In our present study, we explored the clinical manifestations and longterm prognosis of a relatively large cohort of patients with TA with PAI. The aim of our study was to help clinicians systematically recognize PAI in patients with TA.

\footnotetext{
MATERIALS AND METHODS

Patients. The medical records of 598 consecutive inpatients diagnosed with TA from January 2009 to December 2016 were retrospectively reviewed. Of 194 patients who underwent traditional catheter angiography or computed tomography (CT) of PA, 128 had PAI. The patients' medical records were retrospectively reviewed for demographic data, clinical symptoms and signs, comorbidities, laboratory test results, echocardiographic variables, and imaging findings. This research conformed to the ethical guidelines of the 1975 Declaration of Helsinki. The study protocol was approved by the Ethics Committee of Fuwai Hospital (2010-246).
} 
Diagnosis and classification. TA was classified in accordance with the 1990 American College of Rheumatology criteria ${ }^{14}$. All patients fulfilled at least 3 of the following 6 criteria: age of $<40$ years at onset, claudication of the extremities, a decreased brachial artery pulse, a blood pressure difference of $>10 \mathrm{mmHg}$, bruit over the subclavian arteries or aorta, and arteriogram abnormalities $^{14}$. The subtypes of systemic artery involvement were classified into 5 types (I, IIa, IIb, III, IV, and V) based on the classification proposed by Hata, et $a l^{15}$.

PAI was defined as the presence of vascular involvement manifested as stenosis, occlusion, dilation, or aneurysm formation in either PA. For patients who underwent CT pulmonary angiography, we used the PA Obstruction Index (PAOI) to quantify the degree of PA obstruction ${ }^{16}$. The PAOI can be expressed as $\Sigma(\mathrm{n} \times \mathrm{d}) / 40 \times 100$, where $\mathrm{n}$ is the value of the proximal obstruction in the PA tree equal to the number of segmental branches arising distally (minimum, 1; maximum, 20), and $\mathrm{d}$ is the degree of obstruction (minimum, 0; maximum, 2) ${ }^{16}$. According to routine clinical practice at Fuwai Hospital, each PA imaging examination was systematically assessed by qualified chest radiologists. The results were further reviewed by senior chest radiologists.

All the 194 patients with TA in our study underwent echocardiography by certified echocardiologists. The result of each echocardiographic examination was also systematically reviewed by a senior echocardiologist. According to the European Society of Cardiology/European Respiratory Society guidelines for PH, patients with PAI were considered to have a high probability of $\mathrm{PH}$ if their peak tricuspid regurgitation velocity (TRV) was $3.4 \mathrm{~m} / \mathrm{s}$ with or without the presence of other echo "PH signs"17. Patients with a peak TRV of 2.9-3.4 m/s and additional echo signs suggestive of PH were also considered to have a high probability of $\mathrm{PH}^{17}$. The PA systemic pressure is estimated based on the peak TRV, taking the right atrial pressure into account according to the simplified Bernoulli equation ${ }^{17}$. Based on the echocardiographic measurements, left heart disease (LHD) was considered to be present in patients with left atrial or left ventricular enlargement, left ventricular hypertrophy, moderate or severe left heart valve disease, and a left ventricular ejection fraction of $<50 \%$.

Outcome and followup. The major outcome was all-cause mortality. Patients were followed up by consulting the hospital records or performing the clinic interviews or telephone surveys. The followup duration was defined as the length of time from the first admission to March 2018. For the patients who died during followup, the followup duration was defined as the length of time in months from the first admission to the time of death. Patients who were lost to followup were censored at their last available followup visit.

Statistical analysis. Continuous variables with a normal distribution are presented as mean and SD. The differences between groups were analyzed by Student $t$ test. Continuous variables with a non-normal distribution are expressed as median and interquartile range. Comparisons between groups were performed using the Mann-Whitney U test. Qualitative variables are presented as number (percentage). The chi-square test or Fisher's exact test was used to compare the differences. Survival rates were estimated by the Kaplan-Meier method, and differences between groups were assessed by the log-rank test. Univariate and multivariate Cox proportional hazard regressions were used to detect factors independently associated with mortality. The data analysis was conducted using the SPSS 21.0 statistical package (IBM Corp.). Figures were presented by GraphPad Prism 6.01 (GraphPad Software).

\section{RESULTS}

Demographic data and clinical characteristics between patients with TA with and without PAI. The demographic and clinical characteristics of patients with and without PAI are presented in Table 1. Among the 194 patients included in the current study (185 patients had CT and 54 patients had traditional PA angiography), 65\% (120/185) of the patients were confirmed to have PAI by CT, and $98 \%$ (53/54) were confirmed to have PAI by traditional PA angiography. In total, $66 \%(128 / 194)$ patients were diagnosed as PAI by CT or PA angiography. The mean age and disease duration of TA in the patients with PAI was $39.2 \pm 12.3$ years and 60 (24-156) months, respectively, with no significant difference compared to patients without PAI. Patients with PAI more frequently developed dyspnea $(74.2 \%$ vs $22.7 \%$, p < 0.001$)$, hemoptysis (20.3\% vs $3.0 \%, p=0.001)$, and leg edema $(20.3 \%$ vs $3.0 \%$, $\mathrm{p}=0.001)$. Patients with PAI also had an increased risk of developing $\mathrm{PH}(61.7 \%$ vs $7.6 \%, \mathrm{p}<0.001)$ and were more often classified into New York Heart Association (NYHA) class III/IV (40.6\% vs $19.7 \%, \mathrm{p}=0.003)$. The patients with PAI had fewer systemic ischemia-related symptoms and comorbidities such as dizziness $(18.0 \%$ vs $34.8 \%, \mathrm{p}=0.009)$, limb claudication $(19.5 \%$ vs $39.4 \%, \mathrm{p}=0.003)$, hypertension $(47.7 \%$ vs $77.3 \%, \mathrm{p}<0.001)$, and stroke $(3.9 \%$ vs $15.2 \%$, $\mathrm{p}=0.005)$.

Detailed information regarding systemic artery involvement is shown in Figure 1. Patients with PAI had a lower tendency to have involvement of the renal artery $(30.5 \%$ vs $42.4 \%, \mathrm{p}=0.097)$, iliacofemoral artery $(9.4 \%$ vs $18.2 \%$, $\mathrm{p}=0.078)$, and coronary artery $(6.3 \%$ vs $13.6 \%, \mathrm{p}=0.085)$. Patients with PAI had a higher tendency to be classified as having angiographic type IIa systemic artery involvement (8.6\% vs $1.5 \%, p=0.062$; Table 1 ). However, the differences in the distribution of the involved systemic arteries and angiographic classification were not statistically significant between the 2 groups (all p $>0.05$ ).

Angiography features of PA lesion. Most patients with PAI $(84,65.6 \%)$ had bilateral involvement. Among the other 44 $(34.4 \%)$ patients with unilateral involvement, right and left PAI were present in $32(25.0 \%)$ and $12(9.4 \%)$ patients, respectively. The PA trunk was compensatorily enlarged in the patients with PH. The lobar PA $(97,75.8 \%)$ was the most commonly involved artery, followed by the main PA (63, $49.2 \%)$ and segmental PA $(62,48.4 \%)$. The distribution of the involved PA is shown in Table 2. The right upper lobar branches $(72,56.3 \%)$ were the most frequently involved regions. Multibranch PAI was more common than single-branch involvement ( $89.1 \%$ vs $10.9 \%)$. Stenosis and occlusion were the most frequent type of lesions. Aneurysm formation and in situ thrombosis in the PA were detected in $4(3.1 \%)$ and $5(3.9 \%)$ patients, respectively.

Differences between patients with PAI with and without PH. The comparisons between patients with PAI, with and without PH, are displayed in Table 3 . Respiratory symptoms were the initial chief complaints in 68 patients $(60$ in the $\mathrm{PH}$ group vs 8 in the non-PH group, $\mathrm{p}<0.001)$. Among the other 60 patients without respiratory symptoms as the initial symptom, 27 patients were diagnosed with PAI within the first year of diagnosis of TA (10 in the PH group vs 17 in the non-PH group, $\mathrm{p}=0.419), 7$ in the second to third year ( 0 in the $\mathrm{PH}$ group vs 7 in the non-PH group, $\mathrm{p}=0.086)$, and 26 patients after the third year (17 in the PH group vs 9 in the

$$
\text { Personal non-commercial use only. The Journal of Rheumatology Copyright } \odot \text { 2020. All rights reserved. }
$$


Table 1. Demographic and clinical characteristics between patients with TA with and without PAI.

\begin{tabular}{|c|c|c|c|}
\hline Characteristics & TA with PAI, $\mathrm{n}=128$ & TA without PAI, $\mathrm{n}=66$ & $\mathrm{p}$ \\
\hline Age, yrs & $39.2 \pm 12.3$ & $37.7 \pm 14.8$ & 0.493 \\
\hline Female & $111(86.7)$ & $59(89.4)$ & 0.592 \\
\hline Disease duration of TA, mos & $60(24-156)$ & $66(7-216)$ & 0.757 \\
\hline Hypertension & $61(47.7)$ & $51(77.3)$ & $<0.001$ \\
\hline Hyperlipidemia & $27(21.1)$ & $25(37.9)$ & 0.012 \\
\hline Diabetes & $6(4.7)$ & $3(4.5)$ & $>0.999$ \\
\hline Stroke & $5(3.9)$ & $10(15.2)$ & 0.005 \\
\hline \multicolumn{4}{|l|}{ Symptoms and signs } \\
\hline Fever & $14(10.9)$ & $6(9.1)$ & 0.689 \\
\hline Fatigue & $38(29.7)$ & $22(33.3)$ & 0.603 \\
\hline Dizziness & $23(18.0)$ & $23(34.8)$ & 0.009 \\
\hline Blurred vision & $7(5.5)$ & $5(7.6)$ & 0.564 \\
\hline Limb claudication & $25(19.5)$ & $26(39.4)$ & 0.003 \\
\hline Cough & $26(20.3)$ & $8(12.1)$ & 0.155 \\
\hline Dyspnea on exertion & $95(74.2)$ & $15(22.7)$ & $<0.001$ \\
\hline Chest pain & $22(17.2)$ & $10(15.2)$ & 0.717 \\
\hline Hemoptysis & $26(20.3)$ & $2(3.0)$ & 0.001 \\
\hline Syncope & $11(8.6)$ & $6(9.1)$ & 0.908 \\
\hline Pulselessness & $65(50.8)$ & $36(54.5)$ & 0.619 \\
\hline Asymmetric blood pressure & $90(70.3)$ & $47(71.2)$ & 0.896 \\
\hline Subclavian arteries or aorta bruits & $78(60.9)$ & $37(56.1)$ & 0.521 \\
\hline Lower limbs edema & $26(20.3)$ & $2(3.0)$ & 0.001 \\
\hline \multicolumn{4}{|l|}{ Type of vascular involvement } \\
\hline I & $29(22.7)$ & $18(27.3)$ & 0.477 \\
\hline IIa & $11(8.6)$ & $1(1.5)$ & 0.062 \\
\hline IIb & $11(8.6)$ & $5(7.6)$ & 0.807 \\
\hline III & $6(4.7)$ & $1(1.5)$ & 0.426 \\
\hline IV & $9(7.0)$ & $10(15.2)$ & 0.071 \\
\hline $\mathrm{V}$ & $62(48.4)$ & $31(47.0)$ & 0.846 \\
\hline NYHA III/IV & $52(40.6)$ & $13(19.7)$ & 0.003 \\
\hline $\mathrm{ESR}>20 \mathrm{~mm} / \mathrm{h}$ & $39(30.5)$ & $25(37.9)$ & 0.298 \\
\hline $\mathrm{CRP}>8 \mathrm{mg} / \mathrm{l}$ & $40(31.3)$ & $19(28.8)$ & 0.724 \\
\hline High probability of $\mathrm{PH}$ & $79(61.7)$ & $5(7.6)$ & $<0.001$ \\
\hline
\end{tabular}

Data are presented as mean $\pm \mathrm{SD}$, median (interquartile range), or number (percentage). TA: Takayasu arteritis; PAI: pulmonary artery involvement; NYHA: New York Heart Association; ESR: erythrocyte sedimentation rate; CRP: C-reactive protein; PH: pulmonary hypertension.

non-PH group, $\mathrm{p}=0.668)$. Unsurprisingly, clinical manifestations such as dyspnea (94.9\% vs $40.8 \%, \mathrm{p}<0.001)$, hemoptysis $(26.2 \%$ vs $10.2 \%, \mathrm{p}=0.025)$, leg edema $(26.6 \%$ vs $10.2 \%, \mathrm{p}=0.025)$, respiratory failure $\left(\mathrm{PaO}_{2}<60 \mathrm{mmHg}\right.$, $29.1 \%$ vs $6.1 \%, \mathrm{p}=0.001)$, and NYHA class III/IV $(59.5 \%$ vs $10.2 \%, \mathrm{p}<0.001$ ) were more common among patients with than without $\mathrm{PH}$. In addition, patients with $\mathrm{PH}$ less frequently had an elevated C-reactive protein concentration (reference value, $0-8 \mathrm{mg} / 1 ; 22.8 \%$ vs $44.9 \%, \mathrm{p}=0.009$ ) and erythrocyte sedimentation rate (reference value, $<20 \mathrm{~mm} / \mathrm{h}$; $24.1 \%$ vs $40.8 \%, \mathrm{p}=0.045$ ).

Comparing with the patients without $\mathrm{PH}$, patients with $\mathrm{PH}$ more frequently had bilateral PAI $(84.8 \%$ vs $34.7 \%$, $\mathrm{p}<0.001)$ and a higher PAOI [23 (20-27) vs $10(6-15)$, $\mathrm{p}<0.001)$. The PAOI was strongly correlated with PA systemic pressure $(r=0.570, p<0.001)$ as estimated by echocardiography. The numbers of patients in the $\mathrm{PH}$ group with abnormal right cardiac structure and function were as follows: 57 (72.2\%) had right atrial enlargement, 56 (70.9\%) had right ventricular enlargement, and 29 (36.7\%) had right ventricular systolic dysfunction. Patients with $\mathrm{PH}$ less frequently had left ventricular enlargement ( $8.9 \%$ vs $34.7 \%$, $\mathrm{p}<0.001)$ and significant aortic regurgitation $(5.1 \%$ vs $26.5 \%, \mathrm{p}=0.001$ ) than patients without $\mathrm{PH}$.

When we divided all patients with TA with PAI according to the presence of $\mathrm{PH}$ and LHD, 21 patients were assigned to the non-PH and non-LHD group (Group 1), 28 to the non-PH and LHD group (Group 2), 68 to the PH and non-LHD group (Group 3), and 11 to the PH and LHD group (Group 4).

Treatment. Corticosteroids were administered to 61 (77.2\%) and $42(85.7 \%)$ patients with PAI among those with and without $\mathrm{PH}(\mathrm{p}=0.238)$. Immunosuppressants were additionally prescribed to 14 patients with no significant differences between patients with and without PH (13.9\% vs $6.1 \%, p=0.169)$. No patients in our cohort were treated with biologics. Twenty-five patients $(19.5 \%)$ did not receive

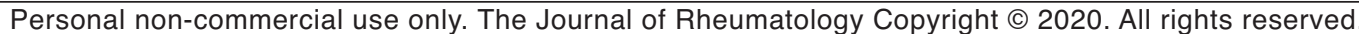




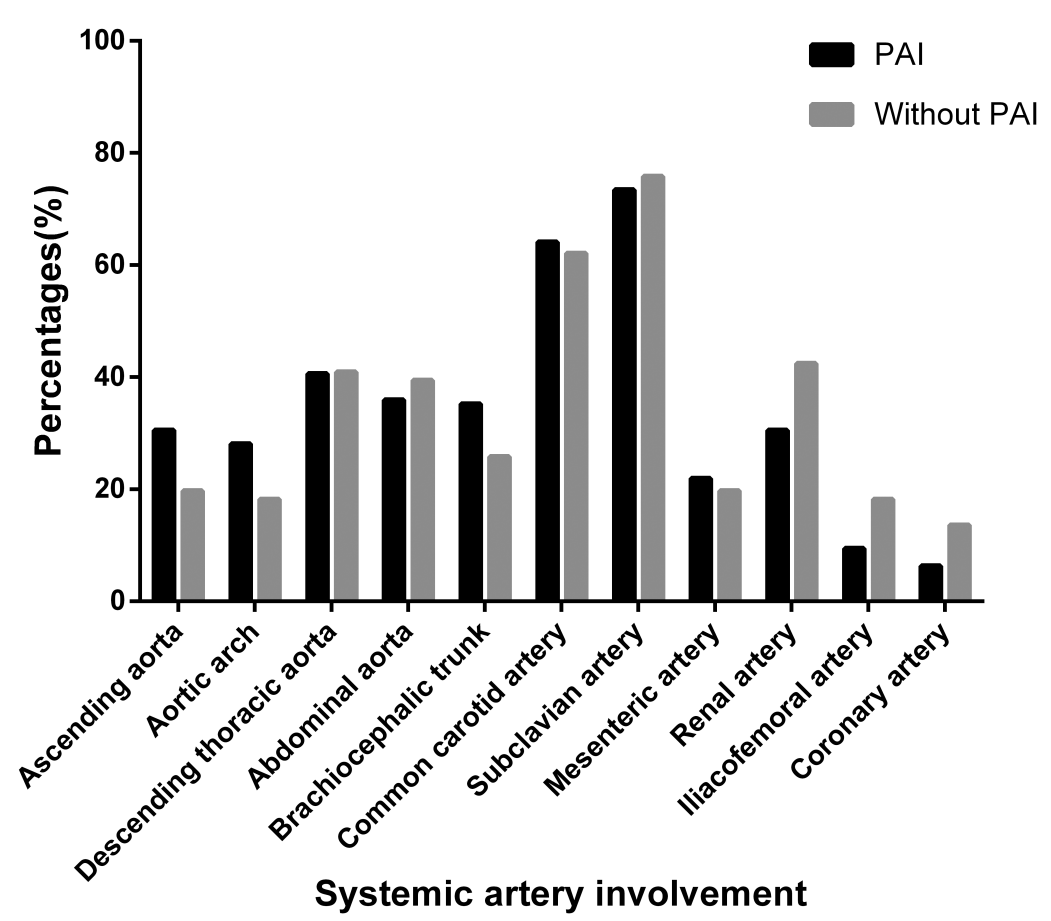

Figure 1. No significant differences in systemic artery involvement were found between patients with and without PAI (all p > 0.05). PAI: pulmonary artery involvement.

Table 2. Angiographic features of PA lesion in 128 TA patients with PAI.

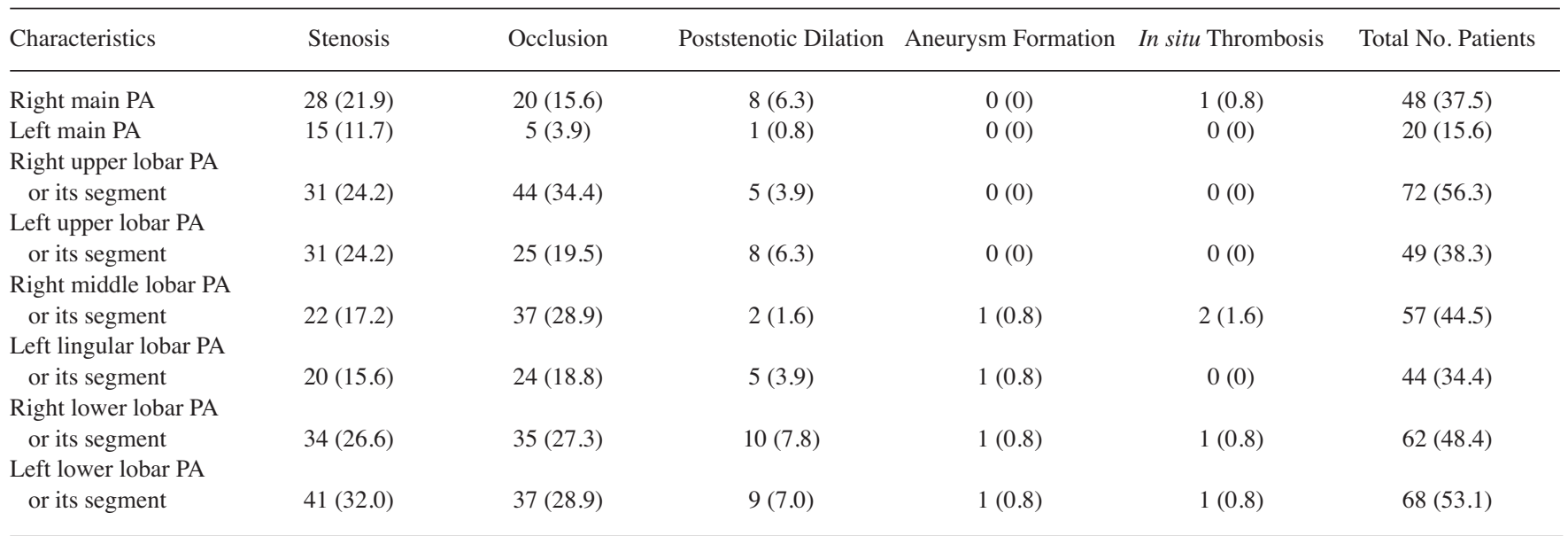

Values are n (\%). PA: pulmonary artery; TA: Takayasu arteritis; PAI: PA involvement.

corticosteroids or immunosuppressive agents because their condition was judged inactive. Among the 79 patients with $\mathrm{PH}, 46$ patients $(58.2 \%)$ were prescribed $\mathrm{PH}$-targeted agents. There were 9 patients with PH-targeted agents and 10 patients without PH-targeted agents who died during followup, and their survival was not significantly improved by the PH-targeted agents $(\mathrm{p}=0.414)$.

Percutaneous transluminal pulmonary angioplasty (PTPA) was performed in 28 patients at the first admission or during followup. The main indication for PTPA was severe PA stenosis combined with severe $\mathrm{PH}$. One patient with occlusion in the right main PA and stenosis of $90 \%$ in the left lower lobar PA died of reperfusion pulmonary edema after PTPA. Among the other 27 patients, the mean PA pressure was decreased from $53 \pm 17$ to $41 \pm 13 \mathrm{mmHg}(\mathrm{p}<0.001)$, and 6 of these patients had a mean PA pressure of $<30 \mathrm{mmHg}$ after PTPA procedure.

Longterm survival and its predictors. During the median followup of 38 (21-58) months, 12 patients were lost to followup and 21 of the remaining 116 patients died. The

$$
\text { Personal non-commercial use only. The Journal of Rheumatology Copyright } \subset \text { (2020. All rights reserved. }
$$


Table 3. Demographic, clinical characteristics, echocardiography, and therapy between patients with PAI with and without $\mathrm{PH}$.

\begin{tabular}{|c|c|c|c|}
\hline Variables & PAI with $\mathrm{PH}, \mathrm{n}=79$ & PAI without $\mathrm{PH}, \mathrm{n}=49$ & $\mathrm{p}$ \\
\hline Age, yrs & $42(30-48)$ & $34(28-44)$ & 0.076 \\
\hline Female & $65(82.3)$ & $46(93.9)$ & 0.067 \\
\hline Onset with respiratory symptoms & $60(75.9)$ & $8(16.3)$ & $<0.001$ \\
\hline Disease duration, mos & $72(24-156)$ & $48(12-162)$ & 0.351 \\
\hline Hypertension & $27(34.2)$ & $34(69.4)$ & $<0.001$ \\
\hline Hyperlipidemia & $13(16.5)$ & $14(28.6)$ & 0.102 \\
\hline Diabetes & $4(5.1)$ & $2(4.1)$ & $>0.999$ \\
\hline Stroke & $2(2.5)$ & $3(6.1)$ & 0.370 \\
\hline \multicolumn{4}{|l|}{ Symptoms and signs } \\
\hline Fever & $7(8.9)$ & $7(14.3)$ & 0.339 \\
\hline Fatigue & $20(25.3)$ & $18(36.7)$ & 0.169 \\
\hline Dizziness & $8(10.1)$ & $15(30.6)$ & 0.003 \\
\hline Blurred vision & $3(3.8)$ & $4(8.2)$ & 0.427 \\
\hline Cough & $20(25.3)$ & $6(12.2)$ & 0.074 \\
\hline Dyspnea on exertion & $75(94.9)$ & $20(40.8)$ & $<0.001$ \\
\hline Chest pain & $13(16.5)$ & $9(18.4)$ & 0.781 \\
\hline Hemoptysis & $21(26.6)$ & $5(10.2)$ & 0.025 \\
\hline Syncope & $6(7.6)$ & $5(10.2)$ & 0.609 \\
\hline Claudication & $9(11.4)$ & $16(32.7)$ & 0.003 \\
\hline Pulselessness & $34(43.0)$ & $31(63.3)$ & 0.026 \\
\hline Asymmetric blood pressure & $51(64.6)$ & $39(79.6)$ & 0.070 \\
\hline Subclavian arteries or aorta bruits & $41(51.9)$ & $37(75.5)$ & 0.008 \\
\hline Lower limbs edema & $21(26.6)$ & $5(10.2)$ & 0.025 \\
\hline NYHA III/IV & $47(59.5)$ & $5(10.2)$ & $<0.001$ \\
\hline Bilateral PA involvement & $67(84.8)$ & $17(34.7)$ & $<0.001$ \\
\hline PAOI & $23(20-27)$ & $10(6-15)$ & $<0.001$ \\
\hline $\mathrm{PaO}_{2}<60 \mathrm{mmHg}$ & $23(29.1)$ & $3(6.1)$ & 0.001 \\
\hline $\mathrm{ESR}>20 \mathrm{~mm} / \mathrm{h}$ & $19(24.1)$ & $20(40.8)$ & 0.045 \\
\hline $\mathrm{CRP}>8 \mathrm{mg} / \mathrm{l}$ & $18(22.8)$ & $22(44.9)$ & 0.009 \\
\hline \multicolumn{4}{|l|}{ Echocardiography } \\
\hline Enlargement of LA & $8(10.1)$ & $9(18.4)$ & 0.182 \\
\hline Enlargement of LV & $7(8.9)$ & $17(34.7)$ & $<0.001$ \\
\hline Enlargement of RA & $57(72.2)$ & 0 & $<0.001$ \\
\hline Enlargement of RV & $56(70.9)$ & 0 & $<0.001$ \\
\hline LV hypertrophy & $7(8.9)$ & $12(24.5)$ & 0.016 \\
\hline Moderate or severe AR & $4(5.1)$ & $13(26.5)$ & 0.001 \\
\hline Moderate or severe MR & $2(2.5)$ & $2(4.1)$ & 0.637 \\
\hline $\mathrm{TRV}, \mathrm{m} / \mathrm{s}$ & $4.4 \pm 0.6$ & $2.7 \pm 0.4$ & $<0.001$ \\
\hline $\mathrm{PASPe}, \mathrm{mmHg}$ & $89.2 \pm 24.5$ & $34.8 \pm 9.9$ & $<0.001$ \\
\hline LVEF $<50 \%$ & $4(5.1)$ & $7(14.3)$ & 0.103 \\
\hline RVSD & $29(36.7)$ & 0 & $<0.001$ \\
\hline PE & $11(13.9)$ & $3(6.1)$ & 0.246 \\
\hline \multicolumn{4}{|l|}{ Therapy } \\
\hline Corticosteroids & $61(77.2)$ & $42(85.7)$ & 0.238 \\
\hline Immunosuppressants & $11(13.9)$ & $3(6.1)$ & 0.169 \\
\hline $\mathrm{PH}$-targeted agents & $46(58.2)$ & 0 & $<0.001$ \\
\hline PTPA & $28(35.4)$ & 0 & $<0.001$ \\
\hline
\end{tabular}

Data are presented as mean $\pm \mathrm{SD}$, median (IQR), or as number (percentage). PAI: pulmonary artery involvement; PH: pulmonary hypertension; NYHA: New York Heart Association; PA: pulmonary artery; PAOI: Pulmonary Artery Obstruction Index; ESR: erythrocyte sedimentation rate; CRP: C-reactive protein; $\mathrm{PaO}_{2}$ : arterial oxygen pressure; LA: left atrium; LV: left ventricular; RA: right atrium; RV: right ventricular; AR: aortic regurgitation; MR: mitral regurgitation; TRV: tricuspid regurgitant velocity; PASPe: estimated pulmonary artery systolic pressure; LVEF: LV ejection fraction; RVSD: RV systolic dysfunction; PE: pericardial effusion; PTPA: percutaneous transluminal pulmonary angioplasty; IQR: interquartile range.

causes of death among the 19 patients with PH were as follows: heart failure in 15 , sudden cardiac death in 2 , massive cerebral infarction in 1 , and post-PTPA reperfusion lung injury in 1 patient. The causes of death in the 2 patients without $\mathrm{PH}$ were severe aortic regurgitation in 1 and severe mitral-aortic regurgitation in 1 . 
The 1-, 3-, and 5-year survival rates in patients with PAI with and without $\mathrm{PH}$ were $93.3 \%$ vs $98.0 \%, 82.3 \%$ vs $98.0 \%$, and $69.0 \%$ vs $92.5 \%$, respectively. Kaplan-Meier analysis showed that patients with PAI with PH had a higher probability of mortality during followup (log-rank test: $p=0.002$; Figure 2A). The patients in the non-PH and non-LHD group (Group 1) had a much better prognosis than the patients with PH (Group 1 vs Group 3, p = 0.012; Group 1 vs Group 4, $\mathrm{p}=0.002$; Figure 2B). Among the 39 patients with LHD, those with PH (Group 4) had a significantly higher mortality rate than those without $\mathrm{PH}$ (Group 2, $\mathrm{p}=0.029$; Figure 2B). Among the 79 patients with $\mathrm{PH}$, no differences were found in mortality between patients with LHD (Group 4) and without LHD (Group 3, p = 0.406; Figure 2B).

In the univariate Cox analyses, the mortality rate was 7-times higher in the patients with PH than without PH (HR $7.003,95 \%$ CI 1.637-30.218, $\mathrm{p}=0.009$ ). The multivariate analyses, which included variables associated with mortality in the univariate analysis, showed that the disease duration (HR 1.004, 95\% CI 1.001-1.007, $\mathrm{p}=0.047$ ), NYHA class III/IV (HR 4.201, 95\% CI 1.269-13.911, p = 0.019), right ventricular systolic dysfunction (HR 3.189, 95\% CI 1.207-8.428, $\mathrm{p}=0.019$ ), and respiratory failure (HR 3.601, 95\% CI 1.416-9.154, $\mathrm{p}=0.007$ ) were independently associated with mortality in patients with TA with PAI (Table 4).

\section{DISCUSSION}

PAI is not rare but the pulmonary circulation is often overlooked in the clinical assessment of TA. According to previous research, when including only patients with TA who have undergone imaging of the PA, the incidence of PAI varied from $33 \%$ to $86 \%$ 3,6,18,19,20,21. In the present study, the ratio of PAI was $66 \%$ among the patients who underwent PA angiography and $21.4 \%$ among all patients with TA, which is consistent with previous studies.
Patients with PAI are more prone to develop PH than those without PAI. Sari, et al ${ }^{12}$ reported that PH was present in $25.0 \%$ of patients with PAI and $7.7 \%$ without PAI. In a case review conducted by Toledano, et $a l^{7}, 42.2 \%$ of patients with PAI also had PH. In the present study, patients with PAI also had a higher risk of developing PH than those without PAI $(61.7 \%$ vs $7.6 \%)$. Mechanical obstruction of the pulmonary vasculature is the leading mechanism underlying the development of PH in TA-associated PAI. Similar to the findings reported by Wang, et $a l^{22}$, the PA pressure was significantly correlated with the extent of PAI in the present study. Additionally, marked reduction in blood flow of the PA leads to hypoxemia because of ventilation-perfusion mismatch. Wang, et $a l^{22}$ also reported that patients with PH due to PAI were more prone to respond to vasoreactivity testing. This suggests that increased vascular tone in the PA may contribute to increases in the PA pressure. Moreover, the coexistence of LHD might contribute to the development of $\mathrm{PH}^{12,22}$, which occurred in 11 of 79 patients with PAI and $\mathrm{PH}$ in our study.

Patients with PH due to TA-associated PAI have a poor prognosis. According to Toledano, et $a l^{7}$, the mortality rate was $20.5 \%$ in patients with PAI and $33.3 \%$ among patients with $\mathrm{PH}$. In a recent Chinese study involving 57 patients with PAI ( 33 with $\mathrm{PH}$ and 24 without $\mathrm{PH}$ ), all 3 patients who died had $\mathrm{PH}^{8}$. In the present study, the risk of mortality was 7 times higher among patients with $\mathrm{PH}$ than among those without $\mathrm{PH}$ during the 38-month followup. Additionally, severe complications of $\mathrm{PH}$ such as right heart failure, respiratory failure, a poor NYHA class, and a long disease duration were independent predictive factors for mortality among patients with TA-associated PAI.

Because of the poor prognosis of $\mathrm{PH}$ in the patients with PAI, pulmonary circulation must be routinely screened in patients with TA in the early stage. Traditional angiography
Figure 2A

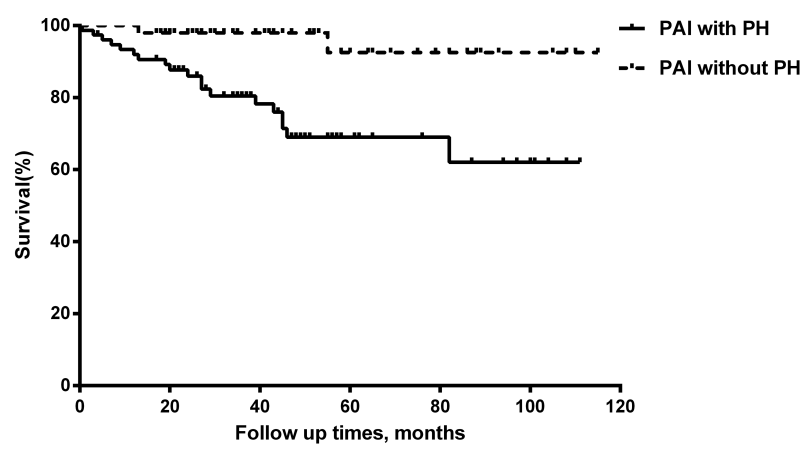

Figure 2B

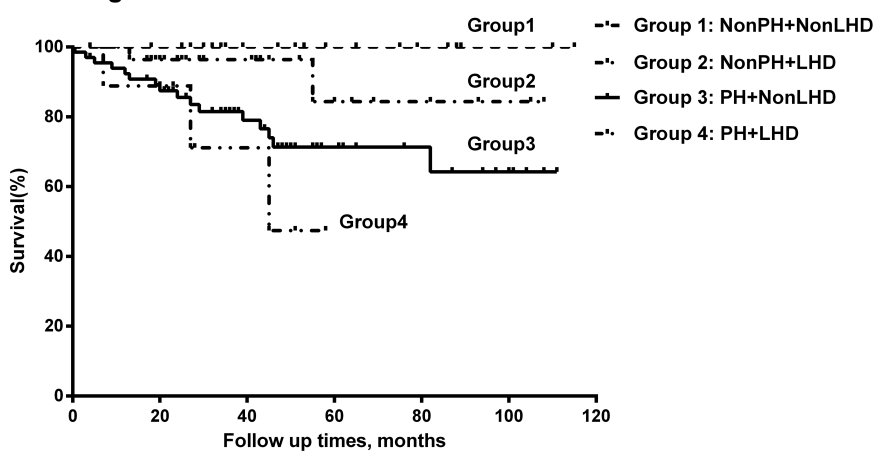

Figure 2. A. Comparison of survival rates between patients with PAI with and without PH. The risk of all-cause mortality was significantly higher in patients with PH ( $\mathrm{p}=0.002)$. B. Comparison of survival rates of patients with PAI on the basis of PH and LHD. Group 1 versus Group 2 (p $=0.161$ ), Group 1 versus Group 3 ( $\mathrm{p}=0.012)$, Group 1 versus Group $4(\mathrm{p}=0.002)$, Group 2 versus Group $4(\mathrm{p}=0.029)$, Group 3 versus Group 4 ( $\mathrm{p}=0.406)$. Group 1: Non-PH and non-LHD; Group 2: Non-PH and LHD; Group 3: PH and non-LHD; Group 4: PH and LHD. PAI: pulmonary artery involvement; PH: pulmonary hypertension; LHD: left heart disease.

Personal non-commercial use only. The Journal of Rheumatology Copyright @ 2020 . All rights reserved. 
Table 4. Predictors of mortality in patients with TA with PAI.

\begin{tabular}{lcccc}
\hline Variables & \multicolumn{2}{c}{ Univariate Analysis } & \multicolumn{2}{c}{ Multivariate Analysis } \\
& HR $(95 \%$ CI $)$ & $\mathrm{p}$ & HR (95\% CI) & $\mathrm{p}$ \\
\hline Age & $1.046(1.010-1.083)$ & 0.011 & & \\
Female & $3.036(0.407-22.633)$ & 0.279 & & \\
Disease duration & $1.005(1.002-1.008)$ & 0.001 & $1.004(1.000-1.007)$ & 0.047 \\
Hypertension & $0.786(0.331-1.866)$ & 0.585 & & \\
Hyperlipidemia & $0.907(0.331-2.483)$ & 0.849 & & \\
Diabetes & $1.487(0.342-6.459)$ & 0.597 & & \\
NYHA class III/IV & $8.072(2.709-24.054)$ & $<0.001$ & $4.201(1.269-13.911)$ & 0.007 \\
Respiratory failure & $4.686(1.939-11.324)$ & 0.001 & $3.601(1.416-9.154)$ & \\
PH & $7.003(1.637-30.218)$ & 0.009 & & 0.019 \\
LHD & $0.834(0.305-2.283)$ & 0.724 & & \\
RVSD & $6.257(2.630-14.885)$ & $<0.001$ & $3.189(1.207-8.428)$ & \\
\end{tabular}

TA: Takayasu arteritis; PAI: pulmonary artery involvement; NYHA: New York Heart Association; PH: pulmonary hypertension; LHD: left heart disease; RVSD: right ventricular systolic dysfunction.

is still the gold standard for detecting lesions in the $\mathrm{PA}^{6,19}$. However, angiography is an invasive procedure and is therefore frequently replaced by $\mathrm{CT}$ and magnetic resonance (MR) angiography, especially in the initial evaluation. CT and MR angiography of the PA can reveal not only stenosis, occlusion, or dilation of the vascular lumen but also thickening of the artery wall ${ }^{8,23,24}$. MR angiography can also disclose early mural inflammatory signs in the PA by late gadolinium enhancement ${ }^{25}$. A more sensitive modality is 18F-fluorodeoxyglucose positron emission tomography (FDG-PET); it can depict active inflammation of the aorta and PA even before any structural change ${ }^{26,27}$. The change in the standardized uptake value with FDG-PET can also help with early evaluation of the treatment response ${ }^{28}$. However, one study showed that FDG-PET may have low power to detect the lesions in small-diameter $\mathrm{PA}^{29}$. Thus, to clearly evaluate lesions and inflammatory activity in the PA, various imaging modalities may be needed to complement each other.

Echocardiography is a simple and noninvasive imaging modality with which to evaluate the structure and function of the heart and estimate the PA pressure. Echocardiography can also provide information on lesions in the right and left main $\mathrm{PA}^{30}$. In the present study, the prognosis of patients with PAI was also well stratified based on the echocardiography assessment. Therefore, echocardiography should be routinely performed for all patients with TA at the time of the initial evaluation, and subsequent assessment is advocated for patients with a high probability of $\mathrm{PH}$ or new-onset symptoms suggesting $\mathrm{PH}$. For patients with a high probability of $\mathrm{PH}$ as evaluated by echocardiography, right heart catheterization is necessary to further assess the hemodynamics and confirm the diagnosis of $\mathrm{PH}^{17}$.

Aggressive stabilization of the disease activity plays a key role in the progression and prognosis of TA-associated PAI and PH. In our cohort, patients with a history of corticosteroid therapy tended to have a lower incidence of $\mathrm{PH}$ than those without history of corticosteroid $(50 \%$ vs $67 \%, p=0.066)$.
Immunosuppressants were less frequently prescribed in our study; thus it was difficult to evaluate their effect on patients with PAI by such limited samples. However, according to Lee, et $l^{31}$, patients with active disease were at high risk of developing PAI and PH. Sari, et al ${ }^{12}$ also reported that patients with severe or treatment-resistant TA had a high risk of PH. Therefore, more widespread use of immunosuppressive agents and biologics might improve the poor prognosis of patients with PAI and PH. PH-targeted drugs were also used but not associated with improvement of survival in our study. However, the therapeutic effect of PH-targeted agents should be further evaluated by a well-designed prospective randomized study and the endpoint should include not only survival but also change in the exercise capacity.

Established PH and stenosis of the PA cannot usually be reversed by medical treatment alone. Pulmonary revascularization is a complementary strategy to restore pulmonary blood flow and should be performed during the inactive disease stage ${ }^{10}$. Surgical treatments seemed to effectively recover the cardiopulmonary function but preclude wide-spread application because of high operational difficulty and surgery-related complications ${ }^{32}$. Inspired by developments in the treatment of chronic thromboembolic $\mathrm{PH}^{33}$, PTPA has also reportedly been applied in single cases and small series of patients with TA-associated PAI. In the present study, the PA pressure decreased after PTPA, but 1 patient who underwent the procedure died of PTPA-associated reperfusion pulmonary edema. Similarly, according to previous studies conducted by Dong, et $a l^{10}$ and Yanagisawa, et $a l^{34}$, although the therapeutic effect of PTPA on TA-associated PAI and $\mathrm{PH}$ was smaller than the effect on chronic thromboembolic PH, the symptoms and hemodynamics were still markedly improved by PTPA. Procedure-related complications such as pulmonary artery injury or reperfusion pulmonary edema were also acceptable ${ }^{10,34}$. Therefore, PTPA is a potential useful therapeutic strategy for patients with $\mathrm{PH}$

Personal non-commercial use only. The Journal of Rheumatology Copyright $@$ 2020. All rights reserved 
because of TA-associated PAI, but it should be performed cautiously. Future case-control studies are required to evaluate the longterm clinical efficacy of PTPA in patients with PAI-associated PH.

The major limitation of our study is that the diagnosis of PH was mainly dependent upon echocardiography. According to current guidelines, right heart catheterization is required to confirm the diagnosis of $\mathrm{PH}$ and identify pre- or postcapillary $\mathrm{PH}$. However, right heart catheterization was difficult to perform on every patient with TA with PAI. Second, the study was conducted in a single institution specializing in cardiovascular disease, and only inpatients who underwent PA angiography were included; this might have resulted in selection bias. Finally, the retrospective study design had the limitations inherent to all retrospective studies. A prospective study is needed to investigate the clinical features and prognosis of patients with TA with PAI.

PAI is not rare in patients with TA. PAI is associated with $\mathrm{PH}$ in patients with TA and increases the risk of mortality. Early detection and aggressive treatment of PAI among patients with TA may improve the poor prognosis.

\section{REFERENCES}

1. Kerr GS, Hallahan CW, Giordano J, Leavitt RY, Fauci AS, Rottem M, et al. Takayasu arteritis. Ann Intern Med 1994;120:919-29.

2. Numano F, Kobayashi Y. Takayasu arteritis - beyond pulselessness. Intern Med 1999;38:226-32.

3. Yamato M, Lecky JW, Hiramatsu K, Kohda E. Takayasu arteritis: radiographic and angiographic findings in 59 patients. Radiology 1986;161:329-34.

4. Sharma BK, Jain S, Radotra BD. An autopsy study of Takayasu arteritis in India. Int J Cardiol 1998;66 Suppl 1:S85-90.

5. Hotchi M. Pathological studies on Takayasu arteritis. Heart Vessels Suppl 1992;7:11-7.

6. Lupi E, Sanchez G, Horwitz S, Gutierrez E. Pulmonary artery involvement in Takayasu's arteritis. Chest 1975;67:69-74.

7. Toledano K, Guralnik L, Lorber A, Ofer A, Yigla M, Rozin A, et al. Pulmonary arteries involvement in Takayasu's arteritis: two cases and literature review. Semin Arthritis Rheum 2011;41:461-70.

8. Gong J, Yang Y, Ma Z, Guo X, Wang J, Kuang T, et al. Clinical and imaging manifestations of Takayasu's arteritis with pulmonary hypertension: a retrospective cohort study in China. Int J Cardiol 2018;276:224-9.

9. Qin L, Hong-Liang Z, Zhi-Hong L, Chang-Ming X, Xin-Hai N. Percutaneous transluminal angioplasty and stenting for pulmonary stenosis due to Takayasu's arteritis: clinical outcome and four-year follow-up. Clin Cardiol 2009;32:639-43.

10. Dong H, Jiang X, Peng M, Zou Y, Guan T, Zhang H, et al. Percutaneous transluminal angioplasty for symptomatic pulmonary stenosis in Takayasu arteritis. J Rheumatol 2014;41:1856-62.

11. Karadag B, Kilic H, Duman D, Ongen Z, Vural VA, Yazici H. Takayasu disease with prominent pulmonary artery involvement: confusion with pulmonary disease leading to delayed diagnosis. Mod Rheumatol 2008;18:507-10.

12. Sari A, Sener YZ, Firat E, Armagan B, Erden A, Oksul M, et al. Pulmonary hypertension in Takayasu arteritis. Int J Rheum Dis 2018;21:1634-9.

13. Mathai SC, Ryan JJ. The growing burden of pulmonary hypertension in the modern era: a zebra no more? Circ Cardiovasc Qual Outcomes 2018;11:e004536.
14. Arend WP, Michel BA, Bloch DA, Hunder GG, Calabrese LH, Edworthy SM, et al. The American College of Rheumatology 1990 criteria for the classification of Takayasu arteritis. Arthritis Rheum 1990;33:1129-34

15. Hata A, Noda M, Moriwaki R, Numano F. Angiographic findings of Takayasu arteritis: new classification. Int J Cardiol 1996;54 Suppl:S155-63.

16. Qanadli SD, El Hajjam M, Vieillard-Baron A, Joseph T, Mesurolle B, Oliva VL, et al. New CT index to quantify arterial obstruction in pulmonary embolism: comparison with angiographic index and echocardiography. AJR Am J Roentgenol 2001;176:1415-20.

17. Galie N, Humbert M, Vachiery JL, Gibbs S, Lang I, Torbicki A, et al; ESC Scientific Document Group. 2015 ESC/ERS Guidelines for the diagnosis and treatment of pulmonary hypertension: The Joint Task Force for the Diagnosis and Treatment of Pulmonary Hypertension of the European Society of Cardiology (ESC) and the European Respiratory Society (ERS): Endorsed by: Association for European Paediatric and Congenital Cardiology (AEPC), International Society for Heart and Lung Transplantation (ISHLT). Eur Heart J 2016;37:67-119.

18. Schmidt J, Kermani TA, Bacani AK, Crowson CS, Cooper LT, Matteson EL, et al. Diagnostic features, treatment, and outcomes of Takayasu arteritis in a US cohort of 126 patients. Mayo Clin Proc 2013;88:822-30.

19. Yamada I, Shibuya H, Matsubara O, Umehara I, Makino T, Numano F, et al. Pulmonary artery disease in Takayasu's arteritis: angiographic findings. AJR Am J Roentgenol 1992;159:263-9.

20. Li J, Sun F, Chen Z, Yang Y, Zhao J, Li M, et al. The clinical characteristics of Chinese Takayasu's arteritis patients: a retrospective study of 411 patients over 24 years. Arthritis Res Ther 2017;19:107.

21. Yang L, Zhang H, Jiang X, Zou Y, Qin F, Song L, et al. Clinical manifestations and longterm outcome for patients with Takayasu arteritis in China. J Rheumatol 2014;41:2439-46.

22. Wang X, Dang A, Chen B, Lv N, Liu Q. Takayasu arteritis-associated pulmonary hypertension. J Rheumatol 2015;42:495-503

23. Tanigawa K, Eguchi K, Kitamura Y, Kawakami A, Ida H, Yamashita $\mathrm{S}$, et al. Magnetic resonance imaging detection of aortic and pulmonary artery wall thickening in the acute stage of Takayasu arteritis. Improvement of clinical and radiologic findings after steroid therapy. Arthritis Rheum 1992;35:476-80.

24. Jin SA, Lee JH, Park JH, Oh JK, Kim MS, Park YK, et al. Endovascular treatment in a patient with left main coronary and pulmonary arterial stenoses as an initial manifestation of Takayasu's arteritis. Heart Lung Circ 2015;24:e26-30.

25. Nucifora G, Todiere G, De Marchi D, Barison A, Aquaro GD, Lombardi M, et al. Severe involvement of pulmonary arteries in Takayasu arteritis: magnetic resonance imaging. Clin Res Cardiol 2011;100:89-92.

26. Bonilla-Abadia F, Pabon LM, Alvarez AM, Canas CA. Clinical utility of $18 \mathrm{~F}$-fluorodeoxyglucose positron emission tomography/computed tomography in Takayasu arteritis - a report of 3 demonstrative cases. J Rheumatol 2013;40:2099.

27. Kobayashi Y, Ishii K, Oda K, Nariai T, Tanaka Y, Ishiwata K, et al. Aortic wall inflammation due to Takayasu arteritis imaged with 18F-FDG PET coregistered with enhanced CT. J Nucl Med 2005;46:917-22.

28. Kusunose K, Yamada H, Tomita N, Nishio S, Niki T, Yamaguchi K, et al. Serial imaging changes during treatment of Takayasu arteritis with pulmonary artery stenosis. Int J Cardiol 2011;148:e47-50.

29. Addimanda O, Spaggiari L, Pipitone N, Versari A, Pattacini P, Salvarani C. Pulmonary artery involvement in Takayasu arteritis. PET/CT versus CT angiography. Clin Exp Rheumatol 2013;1 Suppl 75:S3-4. 
30. Jiang W, Yang Y, Lv X, Li Y, Ma Z, Li J. Echocardiographic characteristics of pulmonary artery involvement in Takayasu arteritis. Echocardiography 2017;34:340-7.

31. Lee GY, Jang SY, Ko SM, Kim EK, Lee SH, Han H, et al. Cardiovascular manifestations of Takayasu arteritis and their relationship to the disease activity: analysis of 204 Korean patients at a single center. Int J Cardiol 2012;159:14-20.

32. Tyagi S, Mehta V, Kashyap R, Kaul UA. Endovascular stent implantation for severe pulmonary artery stenosis in aortoarteritis (Takayasu's arteritis). Catheter Cardiovasc Interv 2004;61:281-5.
33. Aoki T, Sugimura K, Tatebe S, Miura M, Yamamoto S, Yaoita N, et al. Comprehensive evaluation of the effectiveness and safety of balloon pulmonary angioplasty for inoperable chronic thrombo-embolic pulmonary hypertension: long-term effects and procedure-related complications. Eur Heart J 2017;38:3152-9.

34. Yanagisawa R, Kataoka M, Inami T, Fukuda K, Yoshino H, Satoh T. Intravascular imaging-guided percutaneous transluminal pulmonary angioplasty for peripheral pulmonary stenosis and pulmonary Takayasu arteritis. J Heart Lung Transplant 2016;35:537-40. 